\title{
Prediction of aneurysm location based on pattern of bleed on CT scan
}

\author{
Binod Rajbhandari, Mohan Raj Sharma, Sushil Krishna Shilpakar, Gopal Sedain, Amit Pradhanang, Ram Kumar \\ Shrestha, Prakash Kafle, Dipendra Kumar Shrestha, Pushpa Raj Koirala
}

Department of Neurosurgery, Tribhuvan University Teaching Hospital, Maharajgunj, Kathmandu, Nepal

Correspondence: Prof. Mohan Raj Sharma, MS, Department of Neurosurgery, TUTH

Email: mohanrajsharma@gmail.com

\begin{abstract}
Introduction: Computed tomography $(\mathrm{CT})$ is the "gold standard" for detecting subarachnoid hemorrhage (SAH) and digital subtraction angiography (DSA) for visualizing the vascular pathology. There is some correlation between the pattern (based on Fisher grading) and location of bleed on CT scan and eventual location of cerebral aneurysm(s). Our aim was to assess the correlation between distribution of hemorrhage on the initial CT scan and eventual site of the ruptured aneurysm at our institution.

Methods: This retrospective review of prospectively collected data consisted of 50 patients with SAH over 8 months period. CT scan of patients performed within 72 hours after the ictus with suspected SAH were included in the study. Four neurosurgeons, who had at least three years of experience after being qualified, who were blind to the imaging studies, analyzed and scored independently the quantity and distribution of the hemorrhage on CT. Their prediction of the site of the ruptured aneurysm was recorded and this was correlated with the CT angiography (CTA) and/ or DSA findings.

Results: Overall accuracy of prediction was $71.6 \%$ (68.2-75\%). Parenchymal cerebral hematoma was an excellent predictor for the site of a ruptured aneurysm but was present in only a few cases (16\%). The next valid predictor was blood distribution on CT for ruptured anterior communicating artery (ACom) and middle cerebral artery (MCA) aneurysms (89.4\% and 85\% respectively). Pattern of blood on CT together with CTA/DSA findings was found to be reliable for identifying the ruptured aneurysm in patients with multiple aneurysms in all cases.

Conclusion: The quantity and pattern of the blood on CT is a fairly reliable and quick tool for locating a ruptured MCA or ACom aneurysms. It is not, however, reliable for locating other ruptured aneurysms.

Keywords: Aneurysm; Computed Tomography; Digital Subtraction Angiography; Prediction; Subarachnoid Hemorrhage
\end{abstract}

\section{Introduction}

Aneurysmal subarachnoid hemorrhage (aSAH) is a devastating condition with high rates of morbidity and mortality. Worldwide, the incidence of SAH has remain stable over the last 30 years, at around 6/100 000 patients/ year, but in Finland and Japan the incidence is much greater, (up to 20/100 000). ${ }^{1}$ The rupture of intracranial aneurysm (IA) is the cause of non-traumatic SAH in over $80 \%$ of cases. ${ }^{1-3}$ Immediate diagnosis and early identification of the rupture is vital in the surviving patients so that prompt definitive treatment can be carried out to prevent the risk of re-bleeding, a major cause of mortality. 
Diagnosis is based on the presence of hyperdense extravasated blood in the subarachnoid cisterns on high-resolution cranial CT scan images or presence of xanthochromia of cerebrospinal fluid obtained by lumbar puncture in CT negative patients. ${ }^{4}$ Digital subtraction angiography (DSA) is still the gold standard for detection of ruptured intracranial aneurysms, offering both morphological and dynamic information. ${ }^{4}$ Multi-detector CT angiography (CTA) is nowadays an alternative to DSA as a first imaging modality in localizing the aneurysm. ${ }^{3,5}$ CTA can be performed immediately after routine noncontrast CT. It is an accurate, fast and less invasive technology. The important advantage of CTA is the unique opportunity to reconstruct angiography images in various angles and planes. Magnetic resonance angiography (MRA) with 3D time of flight sequence (3DTOF) is also a non-invasive method without radiation for detecting IAs but is less often done due to longer time duration required to obtain the image. ${ }^{6}$

The sensitivity of CTA in detecting a single ruptured aneurysm is above $95 \%$. However, in multiple aneurysms, the diagnostic value of angiographic features for the ruptured aneurysm has not been established yet. The distribution of hemorrhage, based on the initial CT, predicts the location of an aneurysm in over $80 \%$ of cases. ${ }^{7-9}$ It may be possible to determine the site of the ruptured aneurysm in case of multiple aneurysms based on the distribution of blood on the initial CT, but sensitivity in those studies varied widely from $45 \%{ }^{10}$ to $86 \%{ }^{11}$ The prediction of the site of an aneurysm has been reported with contradictory results in cases of both single aneurysms and multiple aneurysms by others. ${ }^{10-13}$

In patients with multiple aneurysms that cannot be clipped in one surgical session, rapid, accurate identification of the ruptured aneurysm is important. ${ }^{14,15}$ Inaccurate determination of the site of the ruptured aneurysm may result in clipping of the wrong aneurysm. It has been reported that postoperative re-bleeding is usually due to a misinterpretation of the location of the ruptured aneurysm in the presence of multiple aneurysms. ${ }^{12,13}$ In such cases, the radiologist should focus to the area of interest in the brain while doing DSA. This will not only shorten the angiographic procedure but also increase the diagnostic yield. ${ }^{14,16,17}$

Tryfonidis et $\mathrm{al}^{7}$ and Karttunen et $\mathrm{al}^{18}$ showed that the correlation between the distribution of hemorrhage and the location of the aneurysm was over $80 \%$. Tryfonidis et $\mathrm{al}^{7}$ also showed that in all cases of multiple aneurysms the ruptured lesion was correctly localized. This study was carried out to assess whether a combination of a variety of radio-anatomical features on non-contrast $\mathrm{CT}$ scans can successfully predict the location of the ruptured aneurysm in all patterns of SAH.

\section{Methods}

This was the retrospective review of prospectively collected data in a high-volume aneurysm surgery center in Nepal (>35 aneurysms clipped /year for last 3 years). The study was carried out after obtaining approval from the Institutional Review Committee of the Institute of Medicine. The study population consisted of 50 patients admitted with the diagnosis of spontaneous SAH in eight months (October 2017 to May 2018) in the department of Neurosurgery at Tribhuvan University Teaching Hospital. Patients above 16 years of age with spontaneous SAH and those with plain CT scan performed within 72 hours after the ictus were included in the study. Those with traumatic $\mathrm{SAH}$ were excluded. The diagnosis was confirmed by CT (128 slice, Siemens, $5 \mathrm{~mm}$ slice) on admission. All patients underwent an initial cerebral CT angiography and DSA was performed in selected cases when the information obtained from CTA was not adequate. DSA was carried out by using Philips FD 20 angio-suite in all cases. This ensured a uniform level of quality and expertise in all cases. For our study, the CT angiogram and DSA findings were reviewed by the radiologists who were unaware of the initial CT findings.

Fifty-five patients with SAH were admitted in the study period. Five patients were excluded (3 refused further investigations and 2 died before further procedures). Four neurosurgeons (raters) were included in the study for the analysis of the findings of the plain CT scan and they independently predicted the location of the aneurysm before they had the opportunity to see the angiographic findings. This was separately recorded by one of the authors (BR) who was not involved in the prediction. The location of aneurysm was considered final based on the findings on CT angiogram or DSA.

The reviewer looked for and identified various radioanatomical features of SAH such as pattern of bleed (e.g., predominant site and location near major vessel bifurcation), presence of intraparenchymal or intraventricular hematoma, distribution of blood in different cisterns and fissures, presence of aneurysm contour if any, and associated hydrocephalus (Table 1). Following careful analysis of the findings and by considering all radio-anatomical features in each case, the reviewers predicted the location of the ruptured aneurysm. This was then compared with the angiogram report. Multiple aneurysms were analyzed separately. 


\section{Table 1 Features identified on CT Scans}

Distribution of subarachnoid blood/hematoma
Predominant site
Left or right
Basal cisterns or convex surface
Anterior or posterior
Location near major vessel bifurcation
Presence and location of parenchymal hematoma
(high attenuation) $+/-$ infarct (low attenuation)
Presence and location of aneurysm contour
Presence of hydrocephalus

A database was then created in SPSS for Mac 24 (SPSS Inc., Chicago, IL) containing all the earlier-mentioned information and statistical analysis was performed. This included chi-squared test to establish whether the identification of an aneurysm on CT scan images is associated with correct identification of the ruptured aneurysm. We also calculated the sensitivity and positive predictive value (PPV) of CT for the site of rupture.

\section{Results}

Mean age of the patients in our study was $58+12$ years and range was from 38 to 85 years. There was a predilection for female sex. (M: $F=1: 2$ ). Most of the patients were of Fisher grade 3 and World Federation of Neurosurgical Societies (WFNS) scale 1 (Table 2, 3). Angiogram was positive for aneurysm in $90 \%$ of the patients. Time interval between SAH and CT was less than 24 hours in $80 \%$.

\section{Table 2. Fisher Grade of SAH Patients}

Fisher Grade
$\begin{array}{ll}1 & 0 \\ 2 & 9(18 \%) \\ 3 & 31(62 \%) \\ 4 & 10(20 \%)\end{array}$

$\begin{array}{ll}\text { WFNS Scale } & \\ 1 & 36(72 \%) \\ 2 & 7(14 \%) \\ 3 & 2(4 \%) \\ 4 & 2(4 \%) \\ 5 & 3(6 \%)\end{array}$

\section{Table 3. WFNS Scale of SAH Patients}

In five cases angiography revealed no aneurysms, indicating a diagnosis of "non-aneurysmal SAH," otherwise called "peri-mesencephalic SAH," in which no definite source of bleeding can be found and the treatment is conservative. Anterior Communicating Artery Complex was the most common involved (38\%) followed by Middle Cerebral Artery (20\%). (Table 4) 3 .

\section{Table 4 Distribution of aneurysms}

\begin{tabular}{ll} 
Types of aneurysms & $\begin{array}{l}\text { Number of } \\
\text { patients }\end{array}$ \\
$\begin{array}{l}\text { Anterior Communicating Artery } \\
\text { Complex } \\
\text { Middle Cerebral Artery }\end{array}$ & $19(38 \%)$ \\
Right & $10(20 \%)$ \\
$\begin{array}{l}\text { Left } \\
\text { PCom Artery }\end{array}$ & $6(12 \%)$ \\
Right & $4(8 \%)$ \\
Left & $3(6 \%)$ \\
Terminal ICA (Right) & $3(6 \%)$ \\
Posterior Circulation & $1(2 \%)$ \\
Basilar & $3(6 \%)$ \\
Posterior Cerebral Artery & $1(2 \%)$ \\
(Right) & $1(2 \%)$ \\
Posterior Inferior Cerebellar & $1(2 \%)$ \\
Prtery(left) & $6(12 \%)$ \\
Multiple & $5(10 \%)$ \\
\hline
\end{tabular}


Multiple aneurysms were present in 6 patients (12\%). Distribution of aneurysms in multiple aneurysms (14 aneurysms in 6 patients) were as follow:

1. Right ICA and left PCom -1

2. Bilateral MCA and left A1 - 1

3. Right Supra-clinoidal and left PCom -1

4. Bilateral MCA and left PCom -1

5. Bilateral MCA - 2

Considering all the aneurysms (excluding peri-mesencephalic ones) including those in multiple also, total numbers of aneurysms were 53 aneurysms in 45 patients. MCA aneurysms were 16 (30\%), ACom 20 (37\%), ICA 3 (5\%) and PCom $9(16 \%)$. Of 45 patients in which aneurysm were detected, 40 patients were operated. Overall prediction rate (Average of all 4 raters) was $71.6 \%(68.2-75 \%)$ for all the aneurysms (excluding multiple ones) but rate was higher for the ruptured ACom Complex and MCA aneurysms.

Table 5. Diagnostic power of prediction of aneurysms by 4 different raters in patients with single aneurysm

\begin{tabular}{|c|c|c|c|c|c|c|c|c|c|c|}
\hline \multirow[b]{2}{*}{ Site by CT Angio } & \multicolumn{2}{|c|}{ Rater 1} & \multicolumn{2}{|c|}{ Rater 2} & \multicolumn{2}{|c|}{ Rater 3} & \multicolumn{2}{|c|}{ Rater 4} & \multirow[b]{2}{*}{ Sensitivity \% } & \multirow[b]{2}{*}{ PPV } \\
\hline & Sen & PPV & Sen & PPV & Sen & PPV & Sen & PPV & & \\
\hline ACom & 89.4 & 100 & 94.7 & 81.8 & 84.2 & 94.1 & 89.4 & 77.2 & 89.4 & 87.1 \\
\hline Right MCA & 83.3 & 62.5 & 100 & 75 & 100 & 54.4 & 66.7 & 66.7 & 87.5 & 63.6 \\
\hline Left MCA & 100 & 80 & 50 & 50 & 75 & 75 & 100 & 80 & 81.25 & 72.2 \\
\hline Right PCom & 66.7 & 100 & 33.3 & 100 & 33.3 & 100 & 33.3 & 100 & 41.6 & 100 \\
\hline Left PCom & 33.3 & 100 & 33.3 & 50 & 33.3 & 50 & 33.3 & 100 & 33.33 & 66.6 \\
\hline ICA & 100 & 25 & 100 & 33 & 100 & 100 & 0 & 0 & 75 & 23 \\
\hline Posterior & 33 & 33 & 0 & 0 & 66.7 & 50 & 33 & 100 & 33.3 & 44.4 \\
\hline PM & 40 & 50 & 20 & 33 & 60 & 75 & 40 & 66 & 40 & 57.1 \\
\hline
\end{tabular}

ACom: Anterior Communicating, MCA: Middle cerebral artery, PCom: Posterior Communicating, ICA: Internal Carotid Artery, PM: Peri-mesencephalic, Post: Posterior Circulation, Sen: Sensitivity

Nineteen ACom aneurysms were identified by CT Angiogram and DSA as a single aneurysm. (Figure 1) Prediction rate (sensitivity) of ACom aneurysms (Table 5) were $89.4 \%, 94.7 \%, 84.2 \%$ and $89.4 \%$ for 4 different raters and positive predictive values for ACom aneurysm were $100 \%, 77.2 \%, 94.1 \%$ and $77.2 \%$. Overall prediction rate for ACom Aneurysm was $89.4 \%$. In 12 cases only, the correctly ruptured aneurysm was same for all 4 raters but at least 3 raters were correct in almost all cases of ACom Aneurysm (18/19).
Prediction proved to be correct in a high proportion of patients with MCA aneurysms. Overall prediction rate for this location was $85 \%$ and for right and left MCA side, it was 87.5 and $81.25 \%$ respectively. All 4 raters agreed in four out of six right MCA. Similarly, prediction rate for left sided MCA aneurysm was also good (81.25\%). Prediction rate of individual rater were $100 \%, 50 \%, 75 \%$ and $100 \%$. 


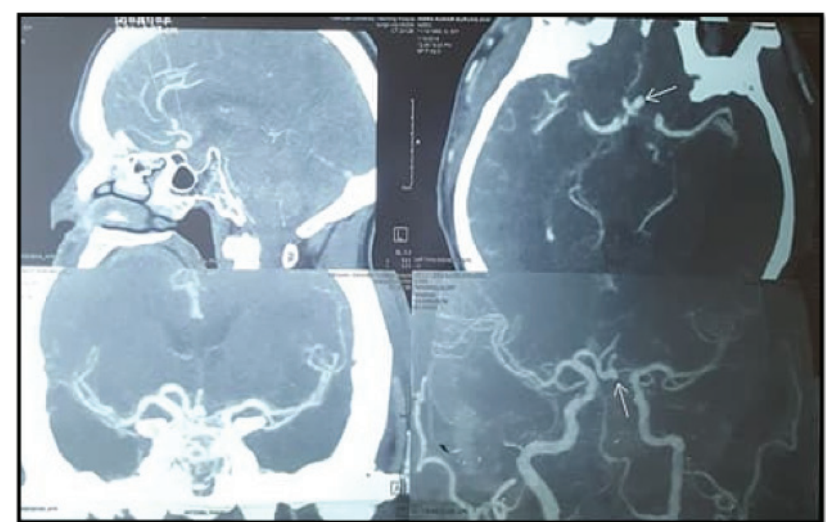

Figure $1 \mathrm{~A}$

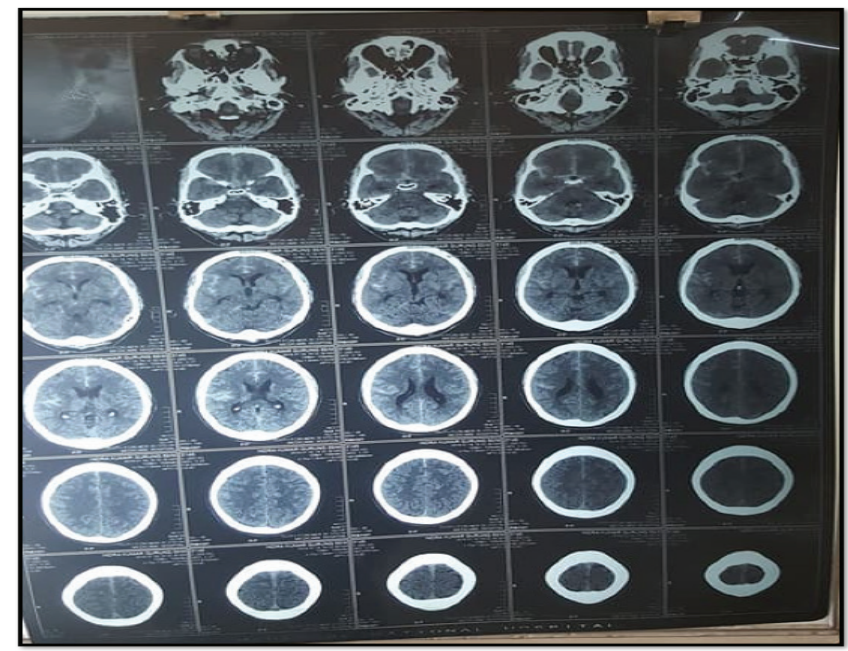

\section{Figure 1 B}

Figure 1. 50-year male with WFNS 1 who underwent clipping on $4^{\text {th }}$ day of ictus. A: Plain CT head showing diffuse SAH. B: CT angiogram showed ACom aneurysm. All 4 raters predicted ACom aneurysm correctly.

The sensitivity of prediction was not satisfactory for other aneurysms. For PCom aneurysms it was only $41.6 \%$ and $33.3 \%$ for right and left sides aneurysms (Overall 37.5\%). 3 raters correctly identified the left ICA aneurysm bleed but there was only one case of ICA aneurysm in our series. Only one rater identified the ruptured basilar and PCA aneurysm whereas none of the raters identified the PICA aneurysm bleed. But only 1 case of each of basilar, PCA and PICA aneurysm were present in out series (Figure 2). Non-aneurysmal SAH was predicted only in two patients by rater 1 , in one patient by rater 2 , in 3 patients by rater 3 and only in 2 patients by rater 4 . (Overall prediction rate only $40 \%$ ). In many patients with a non-aneurysmal hemorrhage, a ruptured ACom or MCA was predicted to have caused the bleed.
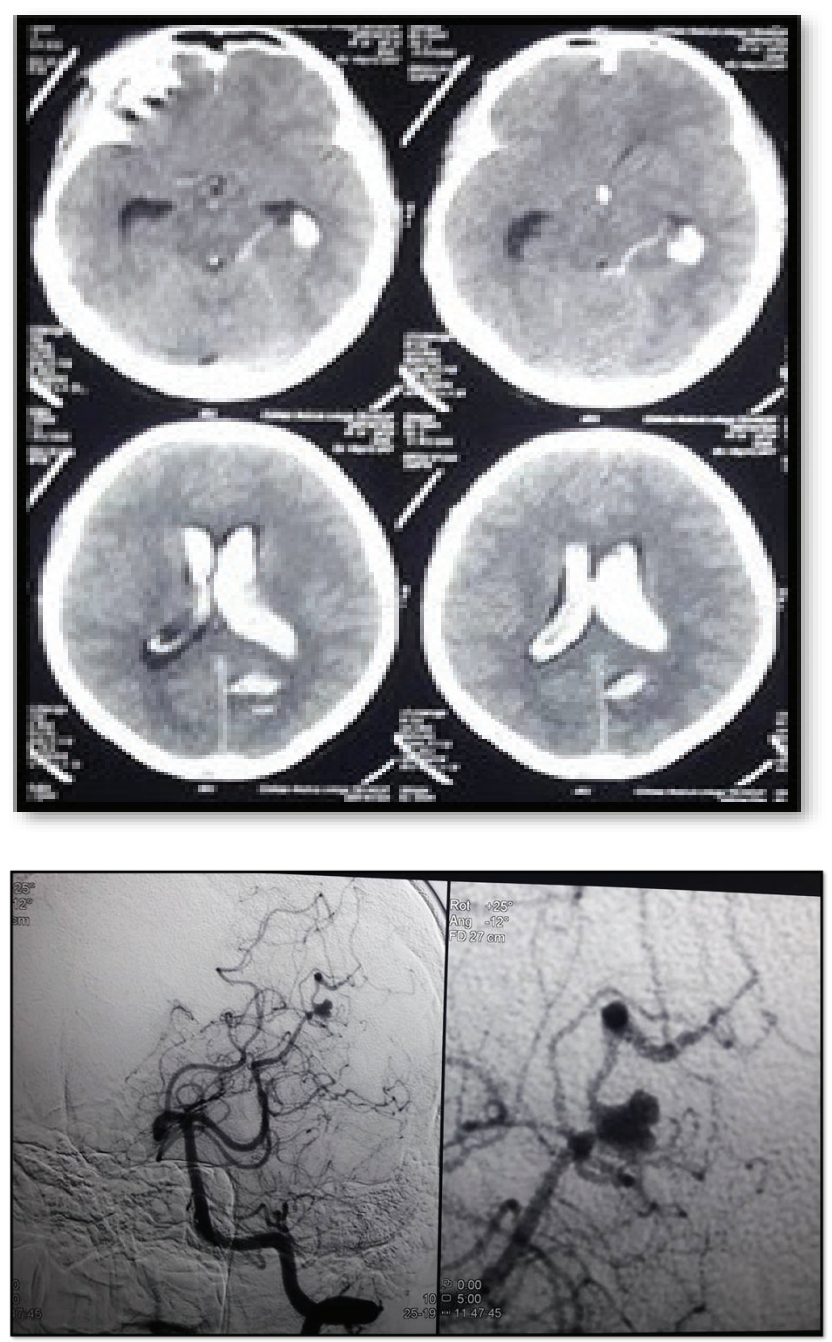

Figure. 228 years/male presented with severe headache. A: CT scan showing blood in lateral and $4^{\text {th }}$ ventricle and parietooccipital region; only one rater predicted the rupture of PCA aneurysm B. DSA showed P4 segment aneurysm bleed who underwent clipping on $16^{\text {th }}$ day

In 6 patients $(12 \%)$, CTA or DSA showed more than one aneurysm. In 4 cases there were 2 and in 2 cases there were 3 aneurysms. An aneurysm was chosen for the operation on the basis of the distribution of the hemorrhage on the initial CT, the irregularity of the outline of the aneurysm, visible aneurysm contour or the existence of the daughter sack of the aneurysm. The neurosurgeon confirmed at operation that the chosen aneurysm was the ruptured one in each patient. In our series there was not a single instance of an unruptured aneurysm operated on instead of the ruptured one, taking the neurosurgeon's opinion as the "gold standard". There were no features on the CT scans to indicate the presence of the remaining non-ruptured aneurysms. 
Parenchymal cerebral hematoma had always occurred in the immediate proximity of the site of rupture but this was present only in $16 \%$ of the patients ( 8 cases) and was excellent predictor of the ruptured site of aneurysm (Overall accuracy 95\%). Presence of ventricular blood and hydrocephalus were not associated with correct localization of the ruptured aneurysm in our series.

\section{Discussion}

Our findings suggest that with careful analysis by experienced neurosurgeons and good knowledge of radio-anatomical features on $\mathrm{CT}$ scans may be a valuable localizing aid for the ruptured aneurysm in SAH. This would be of particular significance in the presence of multiple intracranial aneurysms.

All 4 neurosurgeons estimated the quantity and distribution of the cisternal and intraventricular hemorrhage. However, the method has some drawbacks because of anatomical and technical reasons. The small amount of SAH in the subarachnoid spaces lying next to the skull base may be misread because the slice thickness of CT causes a partial volume effect. The subarachnoid spaces vary in size between patients of different ages, so that the absolute amount of hemorrhage is difficult to estimate. Also, the calcified falx in the interhemispheric fissure may cause difficulties in grading the SAH. Because of these factors, the general visual impression of the amount and distribution of the hemorrhage has a major influence on the final determination of the location of the ruptured aneurysm.

The distribution of aneurysms varies in different reports. ${ }^{7}$ ${ }^{16,18}$ In this study, $37 \%$ of the aneurysms were on the ACom complex, $30 \%$ on the MCA artery, $16 \%$ on the PCom artery and $30 \%$ on the other of intracerebral arteries and in 5 patients out of 50, aneurysm was not detected which correlates to these reports. Posterior circulation aneurysms were seen in $11 \%$ of the total aneurysms. Other distributions have been reported ${ }^{19,20}$. The higher proportion of MCA aneurysms situated on the right side $(56 \%)$ versus the left side (44\%) that we found is comparable to the report of van der Jagt ${ }^{16}$. The reason for this site difference is unknown. Multiple aneurysms were analyzed separately. Considering patients with single aneurysm only, 19 out of $50 \mathrm{SAH}$ patients (38\%) had ACom, 20\% had MCA and $12 \%$ had PCom aneurysms.

In general, the sensitivity and positive predictive value of $\mathrm{CT}$ for the prediction of the site of the ruptured aneurysm in patients with aneurysmal SAH varies widely in other studies (sensitivity from $45 \%$ to $90 \%$ and positive predictive value from $70 \%$ to $100 \% .^{7,16,18}$ In our study, the sensitivity of
CT for the prediction of the site of a ruptured aneurysm depends on the location of the aneurysm. Differentiation by the location of an aneurysm was done in three other studies. $^{8,16,18}$. Sensitivity of predicting ACom and MCA aneurysms were more than $80 \%$ in our study whereas sensitivity was low for other aneurysms. The sensitivity and PPV were almost similar among four neurosurgeons suggesting prediction pattern was similar among the welltrained neurosurgeons. Inter-rater reliability was 0.787 among 4 raters for prediction of aneurysm. The sensitivity values in these studies are similar or higher than they are in our study.

Multiple aneurysms were analyzed separately in our study. In 6 patients out of total $50 \mathrm{SAH}$ patients, multiple aneurysms were seen and were operated on the basis of CT, CTA and/or DSA. In all 6 patients, the ruptured aneurysm was identified correctly. This number is too small to draw a meaningful conclusion. However, this strongly suggests that $\mathrm{CT}$ is of value in predicting the ruptured one in multiple aneurysms. This corroborates with the study by Tryfonidis et $\mathrm{al}^{7}$ where the ruptured lesion was correctly localized in multiple aneurysms.

In this study, the initial $\mathrm{CT}$ proved to be a reliable method for locating ruptured MCA and ACom aneurysms on the basis of the quantity and distribution of SAH. In our small series, we found a strong correlation between predicted and actual ruptured aneurysms. This exceptionally good correlation compared to the previously published reports could be due to small sample size.

SAH associated with parenchymal hematoma (present in only $16 \%$ of cases) had excellent predictability in our study (95\% sensitivity). This finding is consistent with previous studies which consistently report the predictability to be more than $90 \%$. ${ }^{1,16,18}$ Van der Jagt et $\mathrm{al}^{16}$ concluded that the distribution of hemorrhage predicts the location of the aneurysm in over $80 \%$ of cases. ${ }^{7,8,9}$,

As per our findings when an MCA aneurysm bleeds towards the central part of the brain or an ACom aneurysm bleeds towards the lateral parts, there is a possibility of misinterpretation of the location of the ruptured aneurysm, as one or the other neurosurgeons did in 8 instances of ACom and MCA aneurysm rupture.

This is a single center study and has certain limitations. Number of neurosurgeons involved in prediction was heterogenous in terms of years of experience in vascular neurosurgery. In addition, the numbers of predicted ruptured aneurysms were also small. 


\section{Conclusion}

Prediction of presumed site of rupture of aneurysm is very helpful in decision making especially in patients with multiple aneurysms. Plain CT of head is a reliable tool for prediction of ruptured ACom complex and MCA aneurysms. The accuracy of prediction increases when there is associated parenchymal hematoma. CT is not reliable in the prediction of aneurysms in the other locations. A large nationwide study involving neurosurgeons from various centers is recommended to further define the role of plain CT of head in predicting the presumed site of ruptured aneurysm.

\section{References}

1. Van Gijn J, Rinkel GJ. Subarachnoid haemorrhage: diagnosis, causes and management. Brain. 2001;124(Pt 2):249-78. https://doi.org/10.1093/brain/124.2.249; PMid:11157554.

2. Kassell NF, Torner JC, Jane JA, Haley EC, Jr., Adams HP. The International Cooperative Study on the Timing of Aneurysm Surgery. Part 2: Surgical results. J Neurosurg. 1990;73(1):37-47. https://doi.org/10.3171/jns.1990.73.1.0037; PMid:2191091.

3. Velthuis BK, Van Leeuwen MS, Witkamp TD, Ramos LM, Berkelbach van Der Sprenkel JW, Rinkel GJ. Computerized tomography angiography in patients with subarachnoid hemorrhage: from aneurysm detection to treatment without conventional angiography. J Neurosurg. 1999;91(5):761-7.https:// doi.org/10.3171/jns.1999.91.5.0761; PMid:10541232.

4. JMUK-I, Koo B, Trivedi RA, Higgins NJ, Tay KY, Cross JJ, et al. Current diagnostic approaches to subarachnoid haemorrhage. Eur Radiol. 2005;15(6):1135-47. https://doi.org/10.1007/s00330-005-2665-5; PMid:15711840

5. Westerlaan HE, Gravendeel J, Fiore D, Metzemaekers JD, Groen RJ, Mooij JJ, et al. Multislice CT angiography in the selection of patients with ruptured intracranial aneurysms suitable for clipping or coiling. Neuroradiology. 2007;49(12):997-1007. https://doi. org/10.1007/s00234-007-0293-2; $\quad$ PMid:17891387 PMCid:PMC2082066.

6. Kahara VJ, Seppanen SK, Ryymin PS, Mattila P, Kuurne T, Laasonen EM. MR angiography with three-dimensional time-of-flight and targeted maximum-intensity-projection reconstructions in the follow-up of intracranial aneurysms embolized with Guglielmi detachable coils. AJNR Am J Neuroradiol. 1999;20(8):1470-5.; PMid:10512233.

7. Tryfonidis M, Evans AL, Coley SC, Hodgson TL, Connolly DJ, Romanowski CA, et al. The value of radio-anatomical features on non-contrast $\mathrm{CT}$ scans in localizing the source in aneurysmal subarachnoid haemorrhage. Clin Anat. 2007;20(6):618-23. https:// doi.org/10.1002/ca.20475; PMid:17674417.

8. Hillman J. Selective angiography for early aneurysm detection in acute subarachnoid haemorrhage. Acta Neurochir (Wien). 1993;121(1-2):20-5. https://doi. org/10.1007/BF01405178

9. Latchaw RE, Silva P, Falcone SF. The role of CT following aneurysmal rupture. Neuroimaging Clin $\mathrm{N}$ Am. 1997;7(4):693-708. PMid:9336494

10. Nehls DG, Flom RA, Carter LP, Spetzler RF. Multiple intracranial aneurysms: determining the site of rupture. J Neurosurg. 1985;63(3):342-8. https://doi. org/10.3171/jns.1985.63.3.0342; PMid:4020459

11. Kendall BE, Claveria LE. The use of computed axial tomography (CAT) for the diagnosis and management of intracranial angiomas. Neuroradiology. 1976;12(3):141-60. https://doi.org/10.1007/ BF00341859

12. Lee KC, Joo JY, Lee KS. False localization of rupture by computed tomography in bilateral internal carotid artery aneurysms. Surg Neurol. 1996;45(5):43540; discussion 40-1. https://doi.org/10.1016/00903019(95)00409-2

13. Hino A, Fujimoto M, Iwamoto Y, Yamaki T, Katsumori T. False localization of rupture site in patients with multiple cerebral aneurysms and subarachnoid hemorrhage. Neurosurgery. 2000;46(4):825-30. PMid:10764255

14. Rinne J, Hernesniemi J, Niskanen M, Vapalahti M. Management outcome for multiple intracranial aneurysms. Neurosurgery. 1995;36(1):31-7; discussion 7-8. $\quad$ https://doi.org/10.1227/00006123-19950100000003; PMid:7708165

15. Cervoni L, Delfini R, Santoro A, Cantore G. Multiple intracranial aneurysms: surgical treatment and outcome. Acta Neurochir (Wien). 1993;124(2-4):6670. https://doi.org/10.1007/BF01401124 
16. van der Jagt M, Hasan D, Bijvoet HW, Pieterman $H$, Dippel DW, Vermeij FH, et al. Validity of prediction of the site of ruptured intracranial aneurysms with CT. Neurology. 1999;52(1):34-9. https://doi.org/10.1212/ WNL.52.1.34; PMid:9921845.

17. Roos EJ, Rinkel GJ, Velthuis BK, Algra A. The relation between aneurysm size and outcome in patients with subarachnoid hemorrhage. Neurology. 2000;54(12):2334-6. https://doi.org/10.1212/WNL.54.12.2334; PMid:10881266.

18. Karttunen AI, Jartti PH, Ukkola VA, Sajanti J, Haapea M. Value of the quantity and distribution of subarachnoid haemorrhage on CT in the localization of a ruptured cerebral aneurysm. Acta Neurochir (Wien). 2003;145(8):655-61; discussion 61. https://doi. org/10.1007/s00701-003-0080-8; PMid:14520544.

19. Wilson FM, Jaspan T, Holland IM. Multiple cerebral aneurysms--a reappraisal. Neuroradiology. 1989;31(3):232-6. https://doi.org/10.1007/ BF00344349; PMid:2779772.

20. Kopera M, Majchrzak H, Kaspera W. [Prognostic factors in patients with intracerebral hematoma caused by ruptured middle cerebral artery aneurysm]. Neurol Neurochir Pol. 1999;33(2):389-401. PMid:10463253. 